This item is the archived peer-reviewed author-version of:

Structure and function relationship in prolyl oligopeptidase

\title{
Reference:
}

van Elzen Roos, Lambeir Anne-Marie.- Structure and function relationship in prolyl oligopeptidase

CNS and neurological disorders drug targets - ISSN 1871-5273 - 10:3(2011), p. 297-305

Full text (Publisher's DOI): https://doi.org/10.2174/187152711794653814

To cite this reference: https://hdl.handle.net/10067/897010151162165141 


\section{Structure and function relationship in prolyl oligopeptidase}

Roos Van Elzen and Anne-Marie Lambeir

Laboratory of Medical Biochemistry, University of Antwerp - Campus Drie Eiken, Universiteitsplein 1, B-2610

Wilrijk, Belgium

Phone: $+32-3-2652553$

Fax: $+32-3-2652685$

Email: roos.vanelzen@ua.ac.be 


\begin{abstract}
$\underline{\text { Abstract }}$
Prolyl oligopeptidase (POP) belongs to a unique class of serine proteases. Based on extensive enzyme kinetic measurements it has become clear that POP acts in a multifaceted way. This is reflected in the complex behavior in different reaction conditions with different substrates. Also the typical structural architecture of POP, with the active site located at the interface of the catalytic domain and the $\beta$-propeller domain, has instigated many researches to speculate about the mechanism of functioning. The latest developments support the idea of extended conformational changes upon substrate binding. In this review the plethora of available information is assembled into a coherent and stepwise description of the structural composition of POP. In one aspect the composition and contribution of structural boundaries at the active site are described. Attention is focused on the catalytic components and the features that are presumed to confine the substrate specificity. Complementary to this, the specificity of POP towards the residues surrounding the scissile bond is describe by means of a consecutive evaluation of the preferred physico-chemical properties. Together, these two approaches may facilitate a better understanding of the concepts that determine catalytic behavior of POP in physiological conditions.
\end{abstract}

\title{
$\underline{\text { Keywords }}$
}

Prolyl oligopeptidase (POP), peptidase, catalytic triad, active site, oxyanion, substrate specificity

\section{Abbreviations}

POP- Prolyl oligopeptidase; Z- benzyloxycarbonyl; Z-Pro-prolinal- benzyloxycarbonyl-Prolyl-prolinal 


\section{Introduction}

The widespread occurrence, good conservation of prolyl oligopeptidase (POP) and its unique substrate specificity towards prolyl-containing peptides, have led to speculations about its biological importance in physiological and pathophysiological conditions. Especially the capacity of POP to hydrolyze in vitro bioactive peptides, such as oxytocin, substance P, bradykinin, arginine-vasopressin, angiotensins I - IV and many more, at the carboxy-terminal of proline residues, caused much speculation about the biological relevance of POP in neurological and neurodegenerative disorders (reviewed by [1] and [2]). Although the exact mode of action remains an area with many unanswered questions, which designate POP functioning as a puzzling paradox, POP has gained interest from pharmaceutical point of view. This is mainly based on the observed improvement in cognitive functioning upon treatment with a POP inhibitor in a number of animal models [3-8]. Because several proteases, e.g. FAP, might share similar endoproteolytic activity with POP, it is important to design highly specific POP inhibitors in order to further explore this enzyme and its function. Secondly, POP displays only a low specificity. In vitro studies have shown that it can cleave almost any proline containing peptide in nonphysiological situations, although some have reported a preferential order in peptides containing multiple prolines [9]. This, however, does not provide any evidence towards the in vivo actions of POP. In addition, all attempts to elucidate new endogenous substrates in in vivo representative situations, were impeded by methodological and bioinformatical constraints inherently correlated to the unbiased mass spectrometry based exploration of endogenous peptides [9-11]. Thirdly, the interpretation of POP activity measurements, for example in different clinical samples of patients and cell extracts, requires a good understanding of the catalytic behavior in different experimental conditions. The use of different types of substrates can affect POP activity in a different way at different $\mathrm{pH}$ and ionic strength, as described for Z-Gly-Pro- and succinyl-Gly-Pro derivatives [12]. It is likely that such changing behavior is imprinted in the structural architecture of POP.

To this respect major efforts have been done over the years in elucidating and documenting the catalytic and structural properties of POP. There has been a gradual evolution in the understanding which now entails some confusing hypotheses (reviewed by $[13,14]$ ). When reevaluating these reports on the tertiary structure and catalytic mechanism, some themes are recurrently surfaced. For instance, how do peptide substrate and inhibitory ligand access the active site of POP? How is the substrate orientated at the active site of POP, which residues are of importance? Does POP specificity towards substrates reach further than the proline residue at P1? What does the $\mathrm{pH}$-rate dependent catalysis actually mean? When going through these elementary questions it becomes obvious that a thorough understanding of the structural organization of POP, in combination with its functional actions, is not only essential to explain some functional aspects of the catalytic behavior, but also to shed light on the targeted design of specific inhibitors and to reveal crucial aspects of the substrate- specificity. At the very least, the detailed description of the substrate specificity might help in fine-tuning of bioinformatical parameters used to predict new substrates and their cleavage sites.

This review endeavors to assemble the plethora of available information, into a coherent and stepwise description of the structural conformation of POP. On one hand the composition and contribution of structural boundaries at the active site are described. On the other hand, a description is given of the substrate specificity, which is the net result of substrate binding and catalysis. 


\section{POP Structure: a guidance to understanding?}

The major contribution in the structural determination of POP has been provided by the crystal structure of porcine POP in presence of its covalent inhibitor Benzyloxycarbonyl-Prolyl-Prolinal (Z-Pro-prolinal) [15]. With this study, previous predictions made towards the global structural arrangement of POP with a two-domain architecture, the domain motif and the catalytic residues were confirmed (figure 1).

\section{$\underline{2.1 \text { Global structure }}$}

The amino-terminal domain (residues 73-427) is a 7 bladed $\beta$-propeller domain, with each blade built up of 4 anti-parallel strands. The blades are radially arranged, forming a central funnel-like tunnel which connects to a larger side opening formed at the interface of the $\beta$-propeller and the second domain. The catalytic domain, located at the carboxyl terminus, contains a small amino-terminal part (1-72) and a carboxy-terminal part (428710) and structurally resembles the $\alpha, \beta$-hydrolase topology. The domain is assembled by non-covalent interactions between the two regions, although the $\mathrm{N}$-terminal region does not contribute to the $\alpha, \beta$ hydrolase fold nor to the active site. Possibly the main function of residues (1-72) is to provide stability for the circular structure of the $\beta$-propeller by covalently linking it to the peptidase domain [15]. The active site is located in a large cavity at the interface of the peptidase and the $\beta$-propeller domain, and consists of residues from the $\mathrm{C}$ terminal peptidase domain of POP (figure 1 and 2). Based on the linear topology of the three active site residues (serine, aspartate and histidine) and the 3-dimensional conformation of the peptidase domain, POP has been classified as a serine peptidase of the SC clan, family 9.

\section{$\underline{2.2}$ Active site}

The parallelism in organization of the active site between POP and other serine protease families, has provided several clues for the understanding of the structural composition and enzymatic functioning of POP. This has helped in the characterization of the central features necessary for successful hydrolysis of a peptide bond, namely a catalytic triad, a stabilizing oxyanion-binding site, the substrate specificity pocket and surrounding binding sites (figure 2). The latter feature is implicated in the accommodation of the substrate to achieve a catalytically proper orientation in the active site. The unique structure of proline, however, requires special adaptations of the enzyme-substrate-interaction, which is deviant from the classical serine proteases. In most serine proteases, including chymotrypsin and subtilisin, the correct orientation of the substrate and the stabilization of the transition state is established by a S1P $1{ }^{1}$ hydrogen bond [16]. The absence of a main chain $\mathrm{NH}$ group in proline prohibits this H-bond formation and $\beta$-sheet formation between the substrate and the enzyme, which would lead to an incompetent proteolytic activity in the prototypical serine proteases. Due to the specific cleavage pattern of POP for proline containing peptides, it is believed that this S1P1 H-bond is replaced by a hydrogen bond between the carbonyl group of the P2 residue and the guanidium group of Arg643, facilitating hydrolysis after the atypical proline residue [15].

\footnotetext{
${ }^{1}$ According to the formalism introduced by Schechter and Berger, "S" is used for binding sites on the protease and "P" to define amino acid positions of the peptide substrate. The digit "1", in combination with unprimed $\mathrm{P}$ or $\mathrm{S}$, is used for the residue/binding site immediately N-terminal to the scissile bond. The numbers go up moving away from the scissile bond towards the N-terminus. Primed symbols ( $\mathrm{P}^{\prime}$ and $\mathrm{S}$ ' followed by a number) do the same but moving towards the $\mathrm{C}$-terminus.
} 


\section{a. Catalytic triad}

The linear arrangement of the catalytic triad, composed of Ser554, Asp641 and His680, differs from that of the chymotrypsin and subtilisin protease family, but is similar to the $\alpha, \beta$-hydrolases which regroup a diverse group of enzymes such as lipases, cholinesterases, serine carboxypeptidases [17,18]. The catalytic Ser554 is embedded in a well conserved sequence motive -Gly-X-Ser-X-Gly-Gly- (with X any amino acid), common for serine proteases. Moreover, these residues are invariable between 72 aligned enzymes of the POP family [19]. The Ser554 is located at the tip of sharp $\gamma$-like turn, between strand 5 and helix $\mathrm{C}$ of the hydrolase domain ${ }^{2}$, an arrangement referred to as the nucleophile elbow, and is spatially surrounded by several small residues to avoid steric hindrance. Consequently to this, the Ser554 side chain is well exposed and readily accessible to the catalytic imidazole group of His680 on one side, located at a loop between strand 8 and helix F, and to the substrate on the other side. Similar to other serine proteases, the His680 acts as a general base/ acid at different stages of the reaction mechanism. The positioning of the third and acidic residue, Asp641, is directed to allow the coordination of the side chain carboxylate oxygen atoms into a complex hydrogen bond network. One oxygen atom is hydrogen bonded to the in plane oriented imidazole ring of His680 and a well-ordered water molecule. The other oxygen atom is coordinated by two main chain amino groups of Arg643 and Val644 [15]. The analogous orientation and geometry of the three catalytic residues has been an argument for a reaction mechanism similar to that of the prototypical chymotrypsin. This can be summarized as a base/acid driven nucleophilic covalent modification of the non-reactive carbonyl moiety at the scissile bond. In this process, it has been found that the acidic residue functions in a different way between POP and the classical serine proteases. In trypsin, the aspartatic acid participates in the orientation of the correct tautomer of the histidine residue relative to the nucleophilic serine, while in POP, the Asp641 residue is associated with the stabilization of the ion pair between the imidazolium of His680 and the negatively charged tetrahedral intermediate [20]. This was deduced from the observations that in POP the specificity rate constant $\mathrm{k}_{\mathrm{cat}} / \mathrm{K}_{\mathrm{M}}$ between Asp641Asn and Asp641Ala variants decreased to a same extent, and that this change was highly dependent on the substrate employed. A peptide bond is less reactive than the amide bond connecting the chromogenic/fluorogenic leaving groups of synthetic substrates. Hence the contribution of the catalytic triad is more prominent with a normal peptide substrate [20].

Although the catalytic residues are uniformly accepted as the driving force of the hydrolytic reaction, the triad is not the only source of the peptidase activity. This is based on the observation that by disabling of the catalytic triad by one or two residues, the mutant peptidases still hydrolyze substrates three orders of magnitude faster than the non-enzymatic reaction rate. The remaining activity may derive from the free energy of binding, desolvation and the oxyanion binding site (electrophilic catalysis) (reviewed by [21]).

\section{b. The oxyanion binding site}

The oxyanion binding site functions in the stabilization of the tetrahedral intermediates, which occur both in the formation and decomposition of the acylenzyme complex, by hydrogen bonding of the negatively charged oxyanion.

\footnotetext{
${ }^{2}$ The definitions and numbering of the secondary structures are accessible at the PDBsum (e.g. PDB code 1E8N; http://www.ebi.ac.uk/pdbsum/)
} 
While in most hydrolases the two stabilizing hydrogen bonds are established by the main-chain NH group of residues adjacent to the catalytic triad, the topology presents a unique composition in POP and some POP family members. More specifically, this is characterized by the presence of a hydrogen bond between the oxyanion and the hydroxyl group of a tyrosine. In POP the oxyanion binding is provided by the main-chain NH group of Asn555 and the hydroxyl side chain of Tyr473. The latter is situated in a four residue motif YGGF located next to a $\beta$-turn that may assist in the positioning of the residue [15].

The importance of the oxyanion binding site and the Tyr473 hydroxyl group in the catalysis reaction of POP was demonstrated by the destabilization of both the enzyme-substrate complex (increase in $\mathrm{K}_{\mathrm{M}}$ ) and the transition state (decrease in $\mathrm{k}_{\mathrm{cat}}$ ) when using the thiono substrate analogue, Z-Gly-Pro $-\beta$-naphtylamide or a Tyr473Phe variant $[12,22,23]$. Furthermore, the catalytic properties of the Tyr473Phe variant of POP strongly suggest that the hydroxyl group not only operates as an electrophilic catalyst of the reaction, but also participates in the substrate binding. In subtilisin the contribution of the oxyanion binding site is restricted to the transition-state, without affecting the substrate binding. The effects of the oxyanion binding site residue, however, are markedly regulated by the reaction conditions, including type of substrates, $\mathrm{pH}$ and temperature [12,23]. For example less specific $^{3}$ substrates as succinyl-Gly-Pro-nitroanilide are virtually not affected upon damaging of the oxyanion binding site; this is common with the classical serine peptidases. This suggests that, with this type of substrates, the hydrogen bond formation with the oxyanion develops simultaneously with the formation of the transition state. In presence of more specific substrates, however, the binding constant is substantially affected in the truncated enzyme variant. In this case the binding step, which is very likely associated with conformational changes, is more difficult to carry out in absence of the Tyr473 hydroxyl group. Hence, this hydroxyl must interact with the substrate carbonyl group in the ground state of the reaction, not just in transition state only [12].

\section{c. $\quad$ Sub-binding sites}

The $\mathbf{S 1}$ specificity pocket has been designed to ensure a hydrophobic environment and to specifically provide accommodation for a proline residue. It is lined by the side chains of Trp595, Phe476, Val644, Val580 and Tyr599 and the C-atoms of the side chain of Asn555 (table 1, figure 2). Based on the crystal structures of porcine POP the specificity is mainly provided by van der Waals hydrophobic ring stacking between the indole ring of the Trp595 and the pyrrolidine ring of the proline [15,24]. Consequently, the lack of a precise orientation of the P1 residue would adversely affect the enzyme activity as the distance and orientation of the scissile bond carbonyl function becomes improper for a nucleophilic attack by Ser554. This was also evident from the catalytic behavior of Trp595 variants of POP. The substitution of the tryptophan by an alanine resulted in a general impairment of $\mathrm{K}_{\mathrm{M}}$ and $\mathrm{k}_{\mathrm{cat}}$ constants relative to the wild type POP. In Trp595Phe and Tyr variants a slight recovery of the $\mathrm{k}_{\mathrm{cat}}$ constants could be observed. The impact of the substitution on the catalytic parameters were, however, dependent on the substrate, which suggests a non-identical binding mode of the different substrates [25]. This is in contrast an earlier hypothesis that ligands occupy a common binding mode, and that there are no other specific interaction observable between the substrate and the enzyme apart from the S1 pocket. Based on this, and the observations that alteration of the residues surrounding the proline at P1 affects the catalytic parameters, it was suggested that the substrate specificity is determined by substrate-enzyme interactions that occur before the Michaelis complex has formed [26]. In contrast to this, the extensive kinetic

\footnotetext{
${ }^{3}$ In this review the term "specificity" is used to rank substrates according to their $\mathrm{k}_{\text {cat }} / \mathrm{K}_{\mathrm{M}}$ value.
} 
analysis of variants with mutations in the sub-binding sites, suggests that the binding of the substrate might be a more flexible process that does not conform to a rigid single binding mode [25]. Current insights favor a model where initial substrate binding induces a domain movement during which the proline docks in the S1 pocket when the active site is assembled.

The $\mathbf{S 2}$ subsite is less specific for substrate side chains than the S1 pocket. The most important feature is the charged side chain of Arg643 (table 1). The kinetic evaluation of substrates with different P2 residues demonstrates that, at least for small substrates, the P2 side chain residue mainly affects the interaction between the enzyme and substrate backbone. In the crystal structures of POP it has been shown that the substrate P2 side chain interacts with the solvent on one side, while its main carbonyl function tightly interacts with the charged side chain of Arg643. Due to the atypical properties of proline, it is believed that this S2P2 hydrogen bond formation replaces the usually achieved S1P1 interaction, which is essential for the catalysis of most serine proteases. In addition, a second hydrogen bond between the $\mathrm{N} \eta 1$ of Arg643 and the carbonyl at P4 is implicated in the anchoring of the substrate. The catalytic role of the Arg643 residue has been supported by the significant and general impairment of the turnover rate, without reasonably affecting the $\mathrm{K}_{\mathrm{M}}$ constants, upon replacement of the arginine with an alanine residue. Although, still dramatically impaired, the Arg643Lys displayed a tendency to recover these $\mathrm{k}_{\text {cat }}$ values [25]. The lack of a complete restoration of the catalytic properties accords to a spatial distortion of the active site. In the wild type enzyme the Arg643 residue is anchored by the Asp149 through a salt bridge, which supports a well-defined positioning of the guanidium group to allow the establishment of an extensive hydrogen bonding network. This was further illustrated by the dramatic loss in activity with 3 to 4 orders of magnitude upon substituting the equivalent residues in Myxococcus xanthus (Asp145 and Arg618) [27]. It is noteworthy that this salt bridge and hydrogen bond network extends across the interface of the catalytic and the $\beta$-propeller domain. The similarities with the impaired hydrolysis of a substrate with a sulfur substitution at the P2 carbonyl function, and molecular dynamic studies corroborate the hypothesis that such a hydrogen bond network would be implicated in a substrate assisted mechanism of catalysis [22,24,28].

Based on the incapability of the Arg643 variants to hydrolyze substrates with amino acids other than proline at P1, and the described shift in the rate limiting step from acylation to deacylation ${ }^{4}$ in DPP4 catalysis between alanine or proline P1 residue [29], it is argued that the Arg643 has a marked influence on the enzyme acylation.

Further stabilization of the substrate is established by hydrogen bonding between the P3 carbonyl function and the side chain of Trp595. The $\mathbf{S 3}$ subsite itself provides a rather nonpolar environment, being lined by the side chains of several nonpolar residues (Phe173, Met235, Cys255, Ile591 and Ala594) (table 1, figure 2). The fact that a more polar residue at the $\mathrm{P} 3$ position, such as the negatively charged succinyl-Gly-Pro-nitroanilide and free $\alpha$-amino groups, affects the second order rate constant by impairing the $\mathrm{K}_{\mathrm{M}}$ values is due to the weak binding at the hydrophobic S3 subsite $[12,25]$. However, this is not common for all POP sequences, because in many species the POP enzyme contains polar or even charged residues [19].

\footnotetext{
${ }^{4}$ The experimental parameter $\mathrm{k}_{\text {cat }}$ mostly resembles the rate constant of the slowest first order reaction in the catalytic cycle. Differences in leaving group effects and deuterium solvent isotope effects between proline and alanine containing substrates suggest a switch from deacylation to acylation as the rate determining step. However, some caution is advised for the extrapolation of this conclusion to the catalytic behavior of the Arg643 mutants, because a first order step in substrate binding might also become rate limiting. This can be confirmed by measuring deuterium solvent isotope and leaving group effects with the mutant enzymes.
} 
A closer examination of spatially closely positioned Phe173 and Met235, clearly revealed that the S3 pocket lining residues have a different influence on substrate binding and catalysis. The selective impairment of the $K_{M}$ constant in the Phe173Ala variant clearly implies a substrate binding role. Modification of the Met235 residue on the other hand, insinuates a more complex role, given that both $\mathrm{k}_{\text {cat }}$ and $\mathrm{K}_{\mathrm{M}}$ constants are affected in the Met235Ala variant. In addition, the $\mathrm{k}_{\text {cat }}$ recovered considerably to the normal values by the replacement of the methionine for a bulky isoleucine [25].

A third well characterized amino acid at the $\mathbf{S 3}$ subsite is $\mathbf{C y s 2 5 5}$, because it is responsible for the inhibition of porcine POP by bulky thiol reagents. This phenomenon is explained by steric hindrance caused by the close location of the modified thiol group to the S1 subsite, this way excluding substrates from the active site [16] (see figure 2a). POP from Flavobacterium meningosepticum, which is not inhibited by thiol-reagents, has a threonine instead of a Cys255 [30]. In addition, Cys 255 markedly influences the catalytic efficacy at basic $\mathrm{pH}$ values by increasing the substrate affinity [31], which supports a role in the active site and catalysis.

\section{Catalytic mechanism and behavior of POP: aspects of multi-facetted plaver}

Compared to the classical serine proteases, POP acts in an unusual and complex manner depending on the reaction conditions. The most prominent and most described feature entails the $\mathrm{pH}$-sensitive character of POP catalysis. Based on the catalytic mechanism, it is the ionization of the catalytic histidine that governs the $\mathrm{pH}-$ dependence in serine proteases. Consequently the $\mathrm{pH}$-rate profile would resemble a simple titration curve of the histidine residue (pKa 7) in absence of any additional ionizing groups, as is the case for subtilisin. In enzymes of the trypsin family an additional ionizing group with a pKa 9-10 modifies the profile to create a bell-shaped curve. The $\mathrm{pH}$ - dependence of POP, however, displays a more complicated profile which is highly influenced by the type of substrate and environmental conditions including the ionic strength. In presence of the dipeptide substrate Z-Gly-Pro- $\beta$-naphtylamide the profile resembles a doubly shaped curve, which arises from yet another ionization event with an apparent $\mathrm{pKa} \sim 7$, modifying a simple bell shaped curve. This aberrant behavior indicates the existence of two $\mathrm{pH}$-dependent enzyme forms, one dominant at $\mathrm{pH} 6$ and the other at $\mathrm{pH} 8$, that exhibit different catalytic and structural properties, and possibly interconvert with changing $\mathrm{pH}[16,32]$. Possible structural differences between the two $\mathrm{pH}$-dependent enzyme forms have been detected using intrinsic fluorescence measurements, which indicated a more unfolded low-pH form [33].

The differences in solvent isotope effect between the two $\mathrm{pH}$ dependent forms support the idea for a chemical (acylation) based rate-limiting step for the acidic form, while the form operative at physiological $\mathrm{pH}$ would rather be dominated by a physical limited step, e.g. a substrate-binding induced conformational change [32]. For long the actual nature of the presumed physical step has been ambiguous. One theory suggested an oscillating movement of the $\beta$-propeller blades, which might act as a gating filter during catalysis[34]. Molecular dynamics and framework analysis of POP [35], x-ray crystallography of different bacterial variants [36,37] and NMRstudies in presence and absence of the covalent inhibitor Z-Pro-prolinal [38], on the other hand, support the ongoing thesis of substrate-induced inter-domain movements of POP that corresponds to an induced fit mechanism. In this concept, binding of an incoming peptide substrate would shift the equilibrium between the open and closed state in favor of the closed form, leading to a catalytically active conformation of the active site [37]. 
Although this model has been described in bacterial variants of POP only, the high conservation of the active site residues and the residues involved in the inter-domain interactions between different orthologues creates the possibility to extrapolate this mechanism to a more universal context.

\section{Implications on substrate specificity}

Although the analysis of POP structure in complex with different ligands, ranging from a transition-state analogue inhibitor [15], product analogue ligand or a uncleaved octapeptide [24], has provided information about the physico-chemical properties of the active site, this cannot immediately be extrapolated towards the substrate requirements of POP, besides the P1. This is illustrated by the phenomenon that different substrates with significantly altered reaction rate constants and dissimilar $\mathrm{pH}$ dependencies, display an apparent uniform binding to the active site in the crystal structures. These observations suggest that the substrate specificity is not entirely controlled by the binding mode at the active site alone. Instead, it is thought that the substrate specificity of POP is the net result of substrate binding and the rate of catalysis, two processes that are highly dependent on the reaction conditions. By studying the steady-state kinetic constants of POP with different types of substrates, and to a lesser extent by characterizing the cleavage products, some of the specificity characteristics have been revealed.

So far, the described in vitro and in vivo identification and characterization of peptide substrates of POP lack any indication of a hydrolytic activity towards large peptides. In in vivo situations, the average length of identified endogenous peptide substrates of porcine, rat and mouse POP, is between 8 and 20 residues [9-11]. This contributes to the assumption that POP enzymatic activity is restricted to small peptides with an approximate molecular weight of $3000 \mathrm{Da}$ or 30 amino acid residues. It has been hypothesized that this constraint towards large peptide and protein substrates relates to the propensity of polypeptides to assume a 3-dimensional structure $[39,40]$. With the revelation of the crystal structure of POP this restriction has also been interpreted as a so called gating mechanism, where access to the active site is orchestrated by the structural constraints of the POP domains $[41,42]$. Such a mechanism, however, implies that, beyond a certain size, the binding of a substrate to the active site is prohibited. This is somehow conflicting with the recent observations that POP establishes a direct interaction with large protein-like substrates. This limitation of binding to the active site is rather influenced by a combination of several factors including the microenvironment of the substrate and its flexibility, and the conformational movements of the enzyme (e.g. opening and closing). In a recent report indirect evidence was provided suggesting that large, uncleavable substrates can enter the internal cavity and get access to the active site when POP is in an open conformation. This conformation is, however, not compatible with a catalytic competent organization of the active site residues, hampering any hydrolytic activity towards the bound substrate [37].

On the other hand, analyses of $p$-nitrophenylester or $\beta$-naphtylamide peptide derivatives have shown that POP requires a minimum substrate chain length of three residues at the amino-terminal side of the scissile bond $[43,44]$. In POP of mammalian origin, the further lengthening of these synthetic substrate, however, is accompanied with a diminished $\mathrm{k}_{\text {cat }} / \mathrm{K}_{\mathrm{M}}$ value which is mainly attributable to a loss in $\mathrm{k}_{\text {cat }}$. Increasing the chain length on the carboxyl-terminal side of the scissile bond positively influences the $k_{c a t} / K_{M}$ with a 9 fold increase when going from P1' to $\mathrm{P} 2$ ' residue. Addition of a P3' residue reversed this trend to a value comparable to the 
initial ones [44]. In addition and similarly, measurements of the steady-state constants using FRET-peptide substrates showed that increasing the peptide chain length on either side of the scissile bond beyond P3-P3' results in a lower $\mathrm{k}_{\text {cat }} / \mathrm{K}_{\mathrm{M}}$ value, due to an impaired $\mathrm{K}_{\mathrm{M}}$. Conversely, a similar shortening of the substrates is associated with an increase in $\mathrm{K}_{\mathrm{M}}$ which is compensated in part by an improved turnover rate [45]. Hence, it is assumed that the binding site of POP consists of three subsites ( $33, \mathrm{~S} 2$, and $\mathrm{S} 1$ ) at the amino terminus of the scissile bond and two subsites ( $\mathrm{S1}^{\prime}$ and S2') at the carboxyl terminal side that determine the substrate specificity. More recent results suggest that the substrate residues implicated in binding should be extended to at least P4' or even P6' for some peptide substrates [25,27].

Substrate length is only one of many parameters defining the actual specificity. The microenvironment of substrate and enzyme is influenced by the reaction conditions such as $\mathrm{pH}$, ionic strength and temperature.

\section{P1 position}

In accordance to its initial discovery, POP preferentially cleaves a peptide bond that contains a proline residue in the $\mathrm{P} 1$ position, but also other amino acids such as alanine, serine, hydroxyproline, $\alpha$-aminobutyryl and cysteine are accepted $[25,39,46]$ (table 1). Yet, the difference in the $\mathrm{k}_{\mathrm{cat}} / \mathrm{K}_{\mathrm{M}}$ specificity constant covers three orders of magnitude between good and poor substrates, respectively containing a proline and serine in P1 position $[25,46]$. The order of preference towards proteinogenic amino acids is recently established as Pro $>$ Ala - Cys $>$ Ser by analyzing variants of tripeptide AMC (7-amino-4-methylcoumarin) and internally quenched angiotensin II derivatives [25]. This strong specificity of POP toward proline and alanine containing peptides again endorses the presumed relevance in physiological settings.

In addition, POP has an inability to hydrolyze after residues that are thioxylated at the scissile bond of a tetrapeptide [47] or exceed the size and shape of the pyrrolidine ring [46].

\section{P2 position}

In general it is found that POP slightly prefers alanine over glycine at the P2 position. Lysine in this position results in an even higher rate constant but only at low ionic strength; addition of $0.5 \mathrm{M} \mathrm{NaCl}$ results in the same rate constant as for the glycine containing substrate [48]. In agreement to this, positively charged groups (lysine, arginine) in the P2 position are preferred over negatively charged residues (glutamate, aspartate) $[45,49]$. This suggests the presence of some negatively charged moieties near or at the active site of POP that might create an electrostatically favorable environment for the binding of substrates containing a positive charge at P2 [48]. Furthermore, investigation of variable functional groups at the P2 position indicated that POP is able to accommodate amino acids with bulky side chains such as Cys(benzyl), Glu(OMe) [49], and Ser(benzoyl) [50]. This was recently translated in a preference of POP to hydrolyze polar basic amino acids as well as aliphatic branched residues at the P2 position of a 7-mer peptide bradykinin-like fragment [45]. The capacity to accommodate hydrophobic as well as hydrophilic charged residues points toward the presence of an solvent accessible hydrophobic $\mathrm{S} 2$ binding site.

\section{P3 position}

Similarly to the P2 site, the highest $\mathrm{k}_{\mathrm{cat}} / \mathrm{K}_{\mathrm{M}}$ values are obtained for substrates with hydrophobic (leucine and isoleucine) and basic residues (arginine and histidine) on $\mathrm{P} 3$ position, while the presence of negatively charged residues conveys a high stability against POP hydrolysis [45]. In synthetic substrates, this results in a less efficient binding of an amino-terminal succinyl group compared to the benzyloxycarbonyl group; the $\mathrm{K}_{\mathrm{M}}$ values increase considerably with succinyl-Gly-Pro-4-nitroanilide, while the turnover rate remains in the same order of 
magnitude [12]. Moreover, the unusual catalytic behavior of POP with the charged substrate variant in different microenvironmental conditions, suggests the existence of a hydrophobic locus with a nearby positively charged patch that might slow down efficient binding by pulling the substrate away from a catalytically competent position [23]. Such an electrostatic interference might be of relevance in physiological conditions with endogenous substrates.

\section{P1' position}

Apart from one clear constraint of POP against the Pro-Pro bond, all types of amino acid residues are accepted at the P1' position, although with a slight variation in the rate of cleavage [51]. Based on the reaction rate constants and the efficiency of acyl transfer to various nucleophiles, POP reacts the fastest with aromatic (Tyr, Phe) and hydrophobic (Leu) amino acid residues at the P1 vicinal position $[45,51,52]$. The influence of polar and charged residues in this position is more complex and contradictory. Nonetheless there are several indications that introduction of polar and charge residues at the P1' locus is associated with the lowest reactivity of POP [52], especially negatively charged residues would results in poor cleavage rates $[51,53]$. In a more recent report however, this trend could not be confirmed unequivocally, as the $\mathrm{k}_{\text {cat }} / \mathrm{K}_{\mathrm{M}}$ of a glutamate containing FRETpeptide presented the second best value within a set of 13 variable residues at the P1' site [45]. The latter observation is in accordance with an increased susceptibility to proteolysis by POP of a phosphoserine at P1' containing substrate [54] (table 1).

\section{P2' position}

Based on measurements of the acyl transfer to an added nucleophile in Flavobacterium meningosepticum, the P2'-S2' interaction is most efficient in presence of nonpolar, hydrophobic amino acids including proline, followed by polar residues. Charged residues impair the reactivity with almost one order in magnitude [52]. In human POP, the order of preference is more heterogeneous with the highest as well as the lowest reactivities measured in presence of hydrophobic residues (isoleucine and phenylalanine resp.). Also remarkable is the enhancement of the $\mathrm{k}_{\mathrm{cat}} / \mathrm{K}_{\mathrm{M}}$ values in presence of basic residues [45].

\section{P3' position}

The selectivity of POP towards P3' positioned residue is in general very poor, which might be an indication of little participation in the interaction with the enzyme [45]. On the other hand, histidine scanning of the P2'-P4' sites of an uncleavable amino-terminal truncated peptide fragment of Humanin (DLP $\downarrow$ VKRRA) has provided evidence for a S4'-P4'selectivity modifying interaction, which was negligible at the S3'-P3' site [25].

\section{Conclusion}

POP's active site is composed out of residues with hydrophobic side chains and nearby basic residues which have an electrostatic effect that modulates the reactivity. Moreover, despite the embedded character of the active site, it is readily accessible to solvent which contributes to the relatively low specificity of POP. Nevertheless POP displays some unexpected physico-chemical preferences that may reside from conformational alterations in the enzyme and/or substrate. For example the increased hydrolytic efficacy towards substrates with polar or phosphorylated residues at $\mathrm{P} 1{ }^{\prime}$ is almost anomalous to the general tendency to only accept hydrophobic residues. Also the incapability to hydrolyze peptide bonds between two proline residues implies a central role of P1' in determining a proper substrate conformation. It is likely that such substrate induced enzymatic variances cooperate in determining the specificity of POP. Now remains the question to what degree this kinetically 
preferred properties are relevant to the physiologically circulating peptides. There is no knowledge about the properties or composition of the P1 proline flanking residues of putative endogenous POP substrates. For instance, a high preference of POP for hydrophobic residues such as phenylalanine at $\mathrm{P} 2$ is of no value if this combination does not occur in nature. On the other hand, apart from the physical proximity of substrate and enzyme, the prevalence of specific sequences might also provide an extra criterion in the selectivity of POP in vivo. 


\section{Acknowledgements}

This work received support from the EU FP7-HEALTH-2007-B, proposal No. 223077 (nEuroPro). We wish to thank David Ruiz-Carrillo (Probiodrug AG) for kindly providing us insight into the latest results regarding the characterization of some active site residues.

\section{Reference List}

[1] Brandt, I.; Scharpe, S.; Lambeir, A. M. Suggested functions for prolyl oligopeptidase: a puzzling paradox. Clin. Chim. Acta 2007, 377 (1-2), 50-61.

[2] Garcia-Horsman, J. A.; Mannisto, P. T.; Venalainen, J. I. On the role of prolyl oligopeptidase in health and disease. Neuropeptides 2007, 41 (1), 1-24.

[3] Miyazaki, A.; Toide, K.; Sasaki, Y.; Ichitani, Y.; Iwasaki, T. Effect of a prolyl endopeptidase inhibitor, JTP-4819, on radial maze performance in hippocampal-lesioned rats. Pharmacol. Biochem. Behav. 1998, $59(2), 361-368$.

[4] Morain, P.; Lestage, P.; De, N. G.; Jochemsen, R.; Robin, J. L.; Guez, D.; Boyer, P. A. S 17092: a prolyl endopeptidase inhibitor as a potential therapeutic drug for memory impairment. Preclinical and clinical studies. CNS. Drug Rev. 2002, 8 (1), 31-52.

[5] Schneider, J. S.; Giardiniere, M.; Morain, P. Effects of the prolyl endopeptidase inhibitor S 17092 on cognitive deficits in chronic low dose MPTP-treated monkeys. Neuropsychopharmacology 2002, 26 (2), 176-182.

[6] Shishido, Y.; Furushiro, M.; Tanabe, S.; Taniguchi, A.; Hashimoto, S.; Yokokura, T.; Shibata, S.; Yamamoto, T.; Watanabe, S. Effect of ZTTA, a prolyl endopeptidase inhibitor, on memory impairment in a passive avoidance test of rats with basal forebrain lesions. Pharm. Res. 1998, 15 (12), 1907-1910.

[7] Toide, K.; Okamiya, K.; Iwamoto, Y.; Kato, T. Effect of a novel prolyl endopeptidase inhibitor, JTP4819 , on prolyl endopeptidase activity and substance P- and arginine-vasopressin-like immunoreactivity in the brains of aged rats. J. Neurochem. 1995, 65 (1), 234-240.

[8] Toide, K.; Shinoda, M.; Fujiwara, T.; Iwamoto, Y. Effect of a novel prolyl endopeptidase inhibitor, JTP-4819, on spatial memory and central cholinergic neurons in aged rats. Pharmacol. Biochem. Behav. 1997, 56 (3), 427-434.

[9] Nolte, W. M.; Tagore, D. M.; Lane, W. S.; Saghatelian, A. Peptidomics of prolyl endopeptidase in the central nervous system. Biochemistry 2009, 48 (50), 11971-11981.

[10] Brandt, I.; De, V. K.; Devreese, B.; Van, B. J.; Van, D. W.; Augustyns, K.; De, M., I; Scharpe, S.; Lambeir, A. M. Search for substrates for prolyl oligopeptidase in porcine brain. Peptides 2005, 26 (12), 2536-2546.

[11] Tenorio-Laranga, J.; Valero, M. L.; Mannisto, P. T.; Sanchez del, P. M.; Garcia-Horsman, J. A. Combination of snap freezing, differential $\mathrm{pH}$ two-dimensional reverse-phase high-performance liquid chromatography, and iTRAQ technology for the peptidomic analysis of the effect of prolyl oligopeptidase inhibition in the rat brain. Anal. Biochem. 2009, 393 (1), 80-87.

[12] Szeltner, Z.; Renner, V.; Polgar, L. Substrate- and pH-dependent contribution of oxyanion binding site to the catalysis of prolyl oligopeptidase, a paradigm of the serine oligopeptidase family. Protein Sci. 2000, 9 (2), 353-360.

[13] Rea, D.; Fulop, V. Structure-function properties of prolyl oligopeptidase family enzymes. Cell Biochem. Biophys. 2006, 44 (3), 349-365. 
[14] Gass, J.; Khosla, C. Prolyl endopeptidases. Cell Mol. Life Sci. 2007, 64 (3), 345-355.

[15] Fulop, V.; Bocskei, Z.; Polgar, L. Prolyl oligopeptidase: an unusual beta-propeller domain regulates proteolysis. Cell 1998, 94 (2), 161-170.

[16] Polgar, L. pH-dependent mechanism in the catalysis of prolyl endopeptidase from pig muscle. Eur. J. Biochem. 1991, 197 (2), 441-447.

[17] Polgar, L. Structural relationship between lipases and peptidases of the prolyl oligopeptidase family. FEBS Lett. 1992, 311 (3), 281-284.

[18] Goossens, F.; De, M., I; Vanhoof, G.; Hendriks, D.; Vriend, G.; Scharpe, S. The purification, characterization and analysis of primary and secondary-structure of prolyl oligopeptidase from human lymphocytes. Evidence that the enzyme belongs to the alpha/beta hydrolase fold family. Eur. J. Biochem. 1995, 233 (2), 432-441.

[19] Venalainen, J. I.; Juvonen, R. O.; Mannisto, P. T. Evolutionary relationships of the prolyl oligopeptidase family enzymes. Eur. J. Biochem. 2004, 271 (13), 2705-2715.

[20] Szeltner, Z.; Rea, D.; Juhasz, T.; Renner, V.; Mucsi, Z.; Orosz, G.; Fulop, V.; Polgar, L. Substratedependent competency of the catalytic triad of prolyl oligopeptidase. J. Biol. Chem. 2002, 277 (47), 44597-44605.

[21] Polgar, L. The catalytic triad of serine peptidases. Cell Mol. Life Sci. 2005, 62 (19-20), 2161-2172.

[22] Polgar, L.; Kollt, E.; Hollosi, M. Prolyl oligopeptidase catalysis. Reactions with thiono substrates reveal substrate-induced conformational change to be the rate-limiting step. FEBS Lett. 1993, 322 (3), 227 230.

[23] Szeltner, Z.; Rea, D.; Renner, V.; Fulop, V.; Polgar, L. Electrostatic effects and binding determinants in the catalysis of prolyl oligopeptidase. Site specific mutagenesis at the oxyanion binding site. J. Biol. Chem. 2002, 277 (45), 42613-42622.

[24] Fulop, V.; Szeltner, Z.; Renner, V.; Polgar, L. Structures of prolyl oligopeptidase substrate/inhibitor complexes. Use of inhibitor binding for titration of the catalytic histidine residue. J. Biol. Chem. 2001, $276(2), 1262-1266$.

[25] Ruiz-Carrillo, D.; Rahfeld, J. U.; Wagner, L.; Jaenckel, N.; Manhart, S.; Wolf, R.; Ludwig, H. H.; Schulz, I.; Demuth, H. U. Kinetic mapping of the human prolyl oligopeptidase active site. Unpublished Work, 2010.

[26] Szeltner, Z.; Rea, D.; Renner, V.; Juliano, L.; Fulop, V.; Polgar, L. Electrostatic environment at the active site of prolyl oligopeptidase is highly influential during substrate binding. J. Biol. Chem. 2003, 278 (49), 48786-48793.

[27] Shan, L.; Mathews, I. I.; Khosla, C. Structural and mechanistic analysis of two prolyl endopeptidases: role of interdomain dynamics in catalysis and specificity. Proc. Natl. Acad. Sci. U. S. A 2005, 102 (10), 3599-3604.

[28] Kaszuba, K.; Rog, T.; St Pierre, J. F.; Mannisto, P. T.; Karttunen, M.; Bunker, A. Molecular dynamics study of prolyl oligopeptidase with inhibitor in binding cavity. SAR QSAR. Environ. Res. 2009, 20 (78), 595-609.

[29] Heins, J.; Welker, P.; Schonlein, C.; Born, I.; Hartrodt, B.; Neubert, K.; Tsuru, D.; Barth, A. Mechanism of proline-specific proteinases: (I) Substrate specificity of dipeptidyl peptidase IV from pig kidney and proline-specific endopeptidase from Flavobacterium meningosepticum. Biochim. Biophys. Acta 1988, 954 (2), 161-169.

[30] Yoshimoto, T.; Walter, R.; Tsuru, D. Proline-specific endopeptidase from Flavobacterium. Purification and properties. J. Biol. Chem. 1980, 255 (10), 4786-4792. 
[31] Szeltner, Z.; Renner, V.; Polgar, L. The noncatalytic beta-propeller domain of prolyl oligopeptidase enhances the catalytic capability of the peptidase domain. J. Biol. Chem. 2000, 275 (20), 15000-15005.

[32] Polgar, L. Prolyl endopeptidase catalysis. A physical rather than a chemical step is rate-limiting. Biochem. J. 1992, 283 ( Pt 3), 647-648.

[33] Polgar, L. Effects of ionic strength on the catalysis and stability of prolyl oligopeptidase. Biochem. J. 1995, 312 ( Pt 1), 267-271.

[34] Fulop, V.; Szeltner, Z.; Polgar, L. Catalysis of serine oligopeptidases is controlled by a gating filter mechanism. EMBO Rep. 2000, 1 (3), 277-281.

[35] Fuxreiter, M.; Magyar, C.; Juhasz, T.; Szeltner, Z.; Polgar, L.; Simon, I. Flexibility of prolyl oligopeptidase: molecular dynamics and molecular framework analysis of the potential substrate pathways. Proteins 2005, 60 (3), 504-512.

[36] Shan, L.; Mathews, I. I.; Khosla, C. Structural and mechanistic analysis of two prolyl endopeptidases: role of interdomain dynamics in catalysis and specificity. Proc. Natl. Acad. Sci. U. S. A 2005, 102 (10), 3599-3604.

[37] Li, M.; Chen, C.; Davies, D. R.; Chiu, T. K. Induced-fit mechanism for prolyl endopeptidase. J. Biol. Chem. 2010, 285 (28), 21487-21495.

[38] Tarrago, T.; Claasen, B.; Kichik, N.; Rodriguez-Mias, R. A.; Gairi, M.; Giralt, E. A cost-effective labeling strategy for the NMR study of large proteins: selective $15 \mathrm{~N}$-labeling of the tryptophan side chains of prolyl oligopeptidase. Chembiochem. 2009, 10 (17), 2736-2739.

[39] Moriyama, A.; Nakanishi, M.; Takenaka, O.; Sasaki, M. Porcine muscle prolyl endopeptidase: limited proteolysis of tryptic peptides from hemoglobin beta-chains at prolyl and alanyl bonds. Biochim. Biophys. Acta 1988, 956 (2), 151-155.

[40] Moriyama, A.; Nakanishi, M.; Sasaki, M. Porcine muscle prolyl endopeptidase and its endogenous substrates. J. Biochem. 1988, 104 (1), 112-117.

[41] Szeltner, Z.; Rea, D.; Juhasz, T.; Renner, V.; Fulop, V.; Polgar, L. Concerted structural changes in the peptidase and the propeller domains of prolyl oligopeptidase are required for substrate binding. J. Mol. Biol. 2004, 340 (3), 627-637.

[42] Fulop, V.; Szeltner, Z.; Polgar, L. Catalysis of serine oligopeptidases is controlled by a gating filter mechanism. EMBO Rep. 2000, 1 (3), 277-281.

[43] Walter, R.; Yoshimoto, T. Postproline cleaving enzyme: kinetic studies of size and stereospecificity of its active site. Biochemistry 1978, 17 (20), 4139-4144.

[44] Yoshimoto, T.; Simmons, W. H.; Kita, T.; Tsuru, D. Post-proline cleaving enzyme from lamb brain. $J$. Biochem. 1981, 90 (2), 325-334.

[45] Gorrao, S. S.; Hemerly, J. P.; Lima, A. R.; Melo, R. L.; Szeltner, Z.; Polgar, L.; Juliano, M. A.; Juliano, L. Fluorescence resonance energy transfer (FRET) peptides and cycloretro-inverso peptides derived from bradykinin as substrates and inhibitors of prolyl oligopeptidase. Peptides 2007, 28 (11), 21462154.

[46] Nomura, K. Specificity of prolyl endopeptidase. FEBS Lett. 1986, 209 (2), 235-237.

[47] Schutkowski, M.; Jakob, M.; Landgraf, G.; Born, I.; Neubert, K.; Fischer, G. Probing substrate backbone function in prolyl oligopeptidase catalysis--large positional effects of peptide bond monothioxylation. Eur. J. Biochem. 1997, 245 (2), 381-385.

[48] Polgar, L. Unusual secondary specificity of prolyl oligopeptidase and the different reactivities of its two forms toward charged substrates. Biochemistry 1992, 31 (33), 7729-7735. 
[49] Noula, C.; Kokotos, G.; Barth, T.; Tzougraki, C. New fluorogenic substrates for the study of secondary specificity of prolyl oligopeptidase. J. Pept. Res. 1997, 49 (1), 46-51.

[50] Kaspari, A.; Diefenthal, T.; Grosche, G.; Schierhorn, A.; Demuth, H. U. Substrates containing phosphorylated residues adjacent to proline decrease the cleavage by proline-specific peptidases. Biochim. Biophys. Acta 1996, 1293 (1), 147-153.

[51] Koida, M.; Walter, R. Post-proline cleaving enzyme. Purification of this endopeptidase by affinity chromatography. J. Biol. Chem. 1976, 251 (23), 7593-7599.

[52] Bordusa, F.; Jakubke, H. D. The specificity of prolyl endopeptidase from Flavobacterium meningoseptum: mapping the $\mathrm{S}^{\prime}$ subsites by positional scanning via acyl transfer. Bioorg. Med. Chem. 1998, 6 (10), 1775-1780.

[53] Szwajcer-Dey, E.; Rasmussen, J.; Meldal, M.; Breddam, K. Proline-specific endopeptidases from microbial sources: isolation of an enzyme from a Xanthomonas sp. J. Bacteriol. 1992, 174 (8), 24542459 .

[54] Rosen, J.; Tomkinson, B.; Pettersson, G.; Zetterqvist, O. A human serine endopeptidase, purified with respect to activity against a peptide with phosphoserine in the P1' position, is apparently identical with prolyl endopeptidase. J. Biol. Chem. 1991, 266 (6), 3827-3834.

\section{Legends}

Figure 1: Ribbon diagram of porcine prolyl oligopeptidase complexed with Abz-Gly-Phe-Gly-Pro-Phe-GlyPhe $\left(\mathrm{NO}_{2}\right)-$ Ala- $\mathrm{NH}_{2}$ (red). The peptidase domain and $\beta$-propeller domain are colored in blue and green respectively. The surface representation of the ligand binding site clearly illustrates its centrally located character. (drawn with MOE 2009.10 (Chemical computing group), from PDB code 1E8N).

Figure 2: Ligand binding site of the inactive Ser554Ala variant of prolyl oligopeptidase in complex with a peptide substrate (green; Abz-Gly-Phe-Gly-Pro-Phe-Gly-Phe $\left(\mathrm{NO}_{2}\right.$ )-Ala- $\mathrm{NH}_{2}$ ). a) Three dimensional illustration of the arrangement and orientation of 6 residues of the bound octapeptide and the main participating residues of the binding site (see also table 1). Hydrogen bonds are indicated by dashed lines. Secondary structures presented in green and blue belong to the $\beta$-propeller and peptidase domain respectively. b) Two-dimensional projection of the ligand binding site. (figures are drawn with $M O E$ from $\mathrm{PDB}$ code $1 \mathrm{E} 8 \mathrm{~N}$ ). 
Table 1: Summarized overview of the structure function properties at the active site of POP. The residues of the binding cavity are presented according their location in S3S3' sub-binding pocket. Active site residues that have been characterized experimentally are indicated with *. The preferred residues or properties at substrate level are correspondingly presented according the P3-P3' sites. The substrate specificity is further subdivided according residues or modifications that are highly, moderately, and lowly preferred $(++,+$ and $+/-)$ and residues that prevent cleavage by POP (-).

\begin{tabular}{|c|c|c|c|c|c|c|c|c|}
\hline \multicolumn{3}{|c|}{ Enzyme Active Site } & \multicolumn{6}{|c|}{ Substrate } \\
\hline \multirow[t]{2}{*}{ Site } & \multirow[t]{2}{*}{ Residue } & \multirow[t]{2}{*}{ Function } & \multirow[t]{2}{*}{ Position } & \multicolumn{4}{|l|}{ Residue } & \multirow[t]{2}{*}{ Stereospecific } \\
\hline & & & & ++ & + & $+/-$ & -- & \\
\hline \multirow[t]{10}{*}{ S1 } & Ser554* & Catalysis - nucleophile & \multirow[t]{10}{*}{$\mathbf{P 1}$} & \multirow[t]{10}{*}{ Pro } & \multirow[t]{10}{*}{ Ala, } & \multirow{10}{*}{$\begin{array}{l}\text { Ser, Cys, Gly, } \\
\text { Hyp, } \\
<\text { size-shape } \\
\text { pyrrolidine }\end{array}$} & & \multirow[t]{10}{*}{$\mathrm{L}$} \\
\hline & Asp641* & $\begin{array}{l}\text { Catalysis- stabilization } \\
\text { His680 - TI }\end{array}$ & & & & & & \\
\hline & His680 & $\begin{array}{l}\text { Catalysis - acid/base } \\
\text { function }\end{array}$ & & & & & & \\
\hline & Trp595* & Positioning of P1 residue & & & & & & \\
\hline & Asn555 & H-bond with P1 & & & & & & \\
\hline & Tyr473* & $\mathrm{H}$ bond with $\mathrm{P} 1$ & & & & & & \\
\hline & Phe476* & Lining & & & & & & \\
\hline & Val644 & Lining & & & & & & \\
\hline & Val580 & Lining & & & & & & \\
\hline & Tyr599 & Lining & & & & & & \\
\hline $\mathbf{S 2}$ & Arg643* & $\begin{array}{l}\text { H-bond with P2 } \\
\text { Salt bridge with Asp149 }\end{array}$ & $\mathbf{P 2}$ & $\begin{array}{l}\text { Nonpolar: } \\
\text { Ala }>\text { Pro }>\text { Phe }>\text { Gly } \\
\text { Lys }(+)\end{array}$ & & $\begin{array}{l}\text { Neg. charged: } \\
\text { Asp, Glu }\end{array}$ & & $\mathrm{L}$, trans \\
\hline \multirow[t]{6}{*}{$\mathbf{S 3}$} & Trp595 & H-bond with P3 & \multirow[t]{6}{*}{$\mathbf{P 3}$} & \multirow{6}{*}{$\begin{array}{l}\text { Hydrophobic: } \\
\text { (Ile, Leu, Z) }\end{array}$} & \multirow{6}{*}{$\begin{array}{l}\text { Basic charged: } \\
\text { (Arg, His) }\end{array}$} & \multirow{6}{*}{$\begin{array}{l}\text { Neg. charged: } \\
\text { (Asp, Glu, } \\
\text { succinyl) }\end{array}$} & & \\
\hline & Phe173* & Lining & & & & & & \\
\hline & Met235* & Lining + catalysis & & & & & & \\
\hline & Cys $255^{*}$ & Lining & & & & & & \\
\hline & Ile591 & Lining & & & & & & \\
\hline & Ala594 & Lining & & & & & & \\
\hline
\end{tabular}




\begin{tabular}{|l|l|l|l|l|l|l|l|l|}
\hline S1' & & P1' & $\begin{array}{l}\text { Hydrophobic: (Phe, } \\
\text { Leu) } \\
\text { Tyr } \\
\text { Phosphorylation }\end{array}$ & Basic charged & $\begin{array}{l}\text { Pro and } \\
\text { derivatives } \\
\text { (Glu) }\end{array}$ & moderate \\
\hline S2' & His680 & H-bond P2' and Nع2 & P2' & $\begin{array}{l}\text { Nonpolar and } \\
\text { hydrophobic } \\
\text { (Pro > Leu, Ile, } \\
\text { Met, Ala, Phe) }\end{array}$ & $\begin{array}{l}\text { Ser, Asn } \\
\text { His }\end{array}$ & Lys > Asp & & no \\
\hline S3' & & & P3' & Pro & $\sim$ & Lys, Gln & & no \\
\hline
\end{tabular}


Figure 1

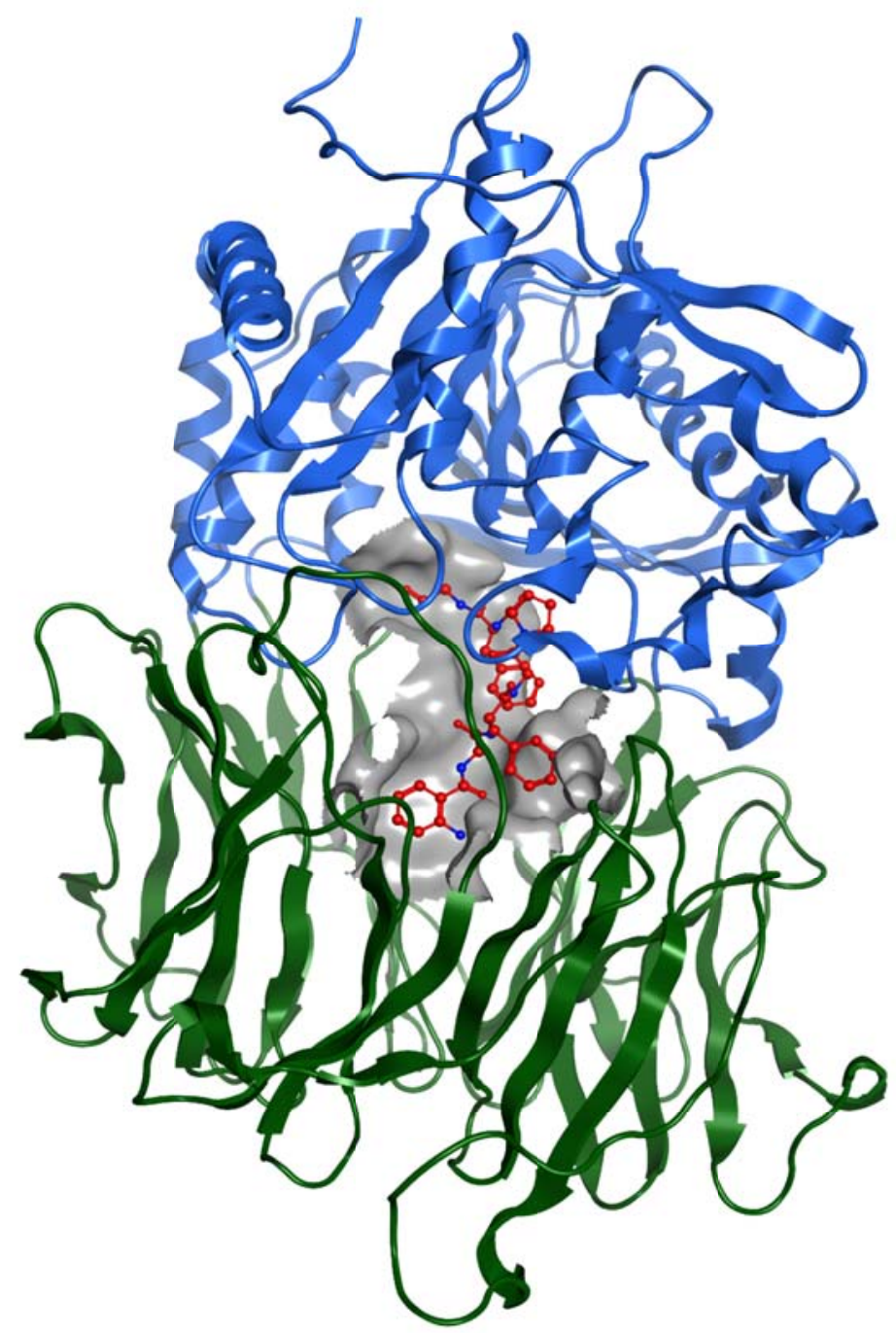


Figure 2

a

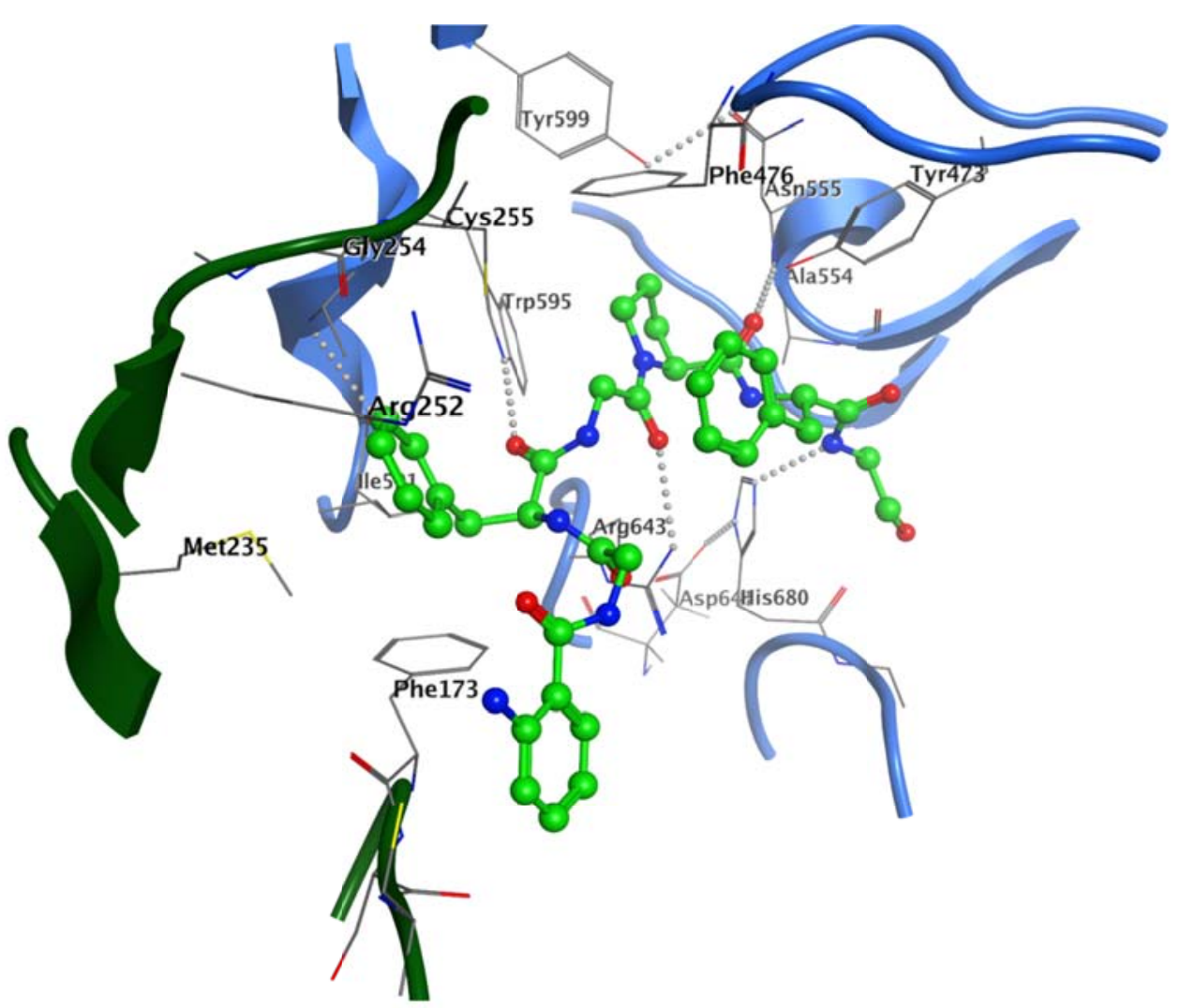

b

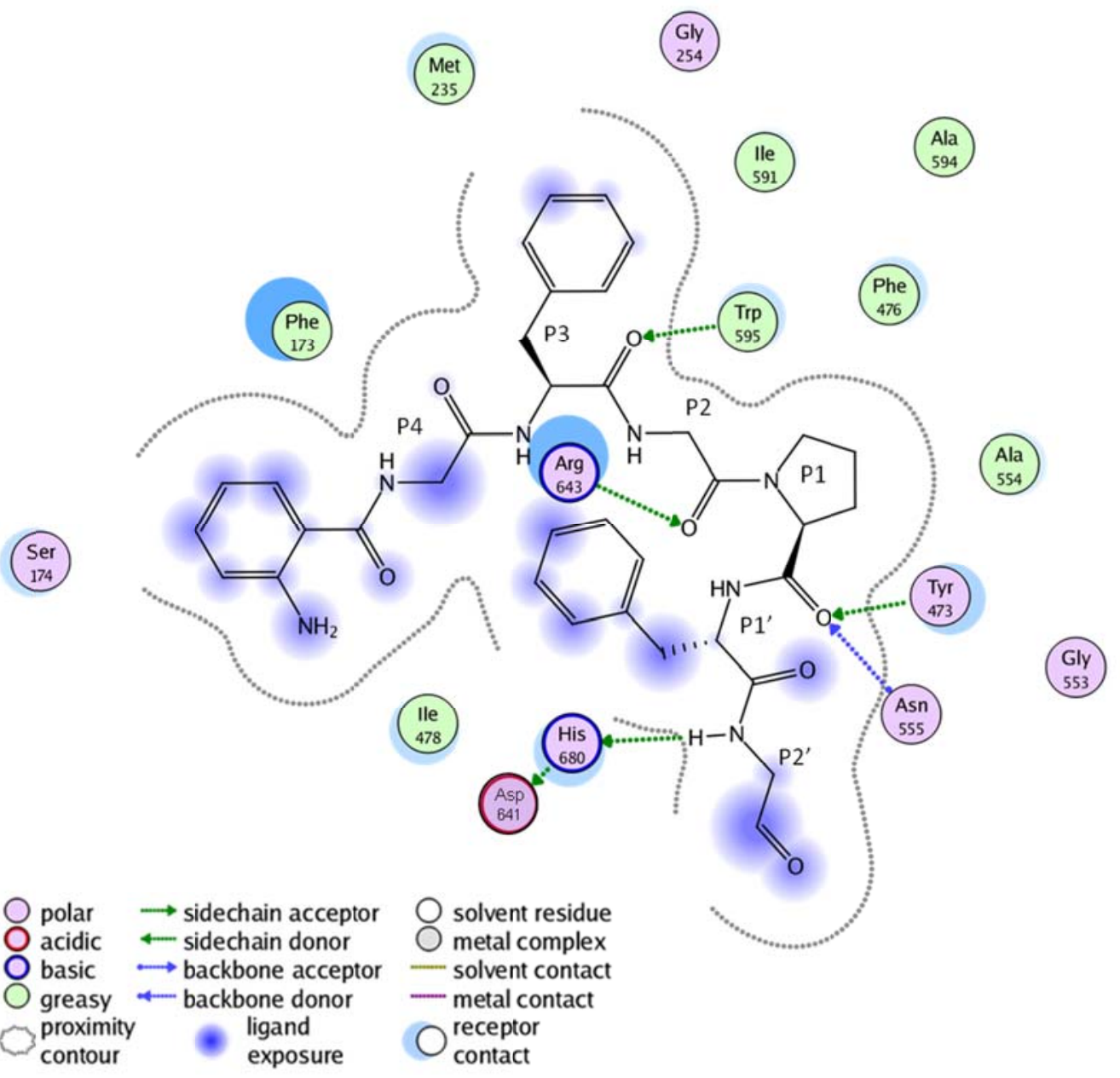

